\title{
Real-World Health Care Costs Based on Medication Adherence and Risk of Stroke and Bleeding in Patients Treated with Novel Anticoagulant Therapy
}

\author{
Chinmay G. Deshpande, BPharm, MS, PhD; Stephen Kogut, PhD, MBA; and Cynthia Willey, PhD
}

\begin{abstract}
BACKGROUND: With the lack of real-world evidence, the challenge for drug reimbursement policy decision makers is to understand medication adherence behavior among users of novel oral anticoagulants (NOACs) and its effect on overall cost savings. No study has examined and quantified the burden of cost in high-risk patients taking NOAC therapy.
\end{abstract}

OBJECTIVE: To examine the association of cost with adherence, comorbidity, and risk of stroke and bleeding in patients taking NOACs (rivaroxaban and dabigatran).

METHODS: A retrospective cohort study used deidentified data from a commercial managed care database affiliated with Optum Clinformatics Data Mart (January 1, 2010-December 31, 2012). Patients aged 18 years and older with $\geq 1$ diagnosis of atrial fibrillation/flutter, $>1$ NOAC prescription, 6-month pre-index and 12-month post-index continuous enrollment, and $\mathrm{CHA}_{2} \mathrm{DS}_{2}$-VASc score $\geq 1$ were included. Adherence was calculated using proportion of days covered ( $\mathrm{PDC} \geq 80 \%$ ) over an assessment period of 3 , 6 , and 12 months and compared based on level of comorbidity, stroke, and bleeding risk. The adjusted annual health care costs per patient (drug, medical, and total) were calculated using multivariable gamma regression controlling for demographic and clinical characteristics and compared across groups based on adherence over 12 months, baseline level of comorbidity, and risk of stroke and bleeding.

RESULTS: Of 25,120 NOAC patients, 2,981 patients were included in the final cohort. Based on a PDC threshold of $\geq 80 \%$, the adherence rate over 3,6 , and 12 months was $72 \%, 65 \%$, and $54 \%$, respectively. For all time periods, the level of adherence significantly increased $(P<0.001)$, with an increase in stroke risk (based on $\mathrm{CHA}_{2} \mathrm{DS}_{2} \mathrm{VASc}$ scores of 1, 2-3, and 4+); comorbidity (Charlson Comorbidity Index scores of $0,1-2$, and $3+$ ); and risk of bleeding (HAS-BLED scores of 0-1, 2, and 3+). Adjusted all-cause total cost calculated for a 12-month period was significantly lower $(\$ 29,742$ vs. $\$ 33,609$ ) among adherent versus nonadherent users. Drug cost was higher $(\$ 5,595$ vs. $\$ 2,233)$ among adherent versus nonadherent patients but was offset by lower medical costs $(\$ 23,544$ vs. $\$ 30,485)$ costs. The overall cost significantly increased for patients with a high risk of bleeding and a high level of comorbidity.

CONCLUSIONS: Adherence to NOAC therapy led to a reduction in overall health care cost, since higher drug costs were offset by lower medical (inpatient and outpatient) costs among adherent patients. Cost information based on adherence and risk of stroke and bleeding can help formulary decision makers to assess risk-benefit and help clinicians in developing interventions to reduce patient burden.

J Manag Care Spec Pharm. 2018;24(5):430-39

Copyright $\odot 2018$, Academy of Managed Care Pharmacy. All rights reserved.

\section{What is already known about this subject}

Novel oral anticoagulants (NOACs) have shown better or similar efficacy in the reduction of stroke risk and noninferior risk of major bleeding versus warfarin in clinical trials.

NOACs have a promising potential to improve medication adherence because of fewer dietary and drug-to-drug interactions, fixed dosage, and the need for minimal monitoring.

\section{What this study adds}

This study provides new evidence based on real-world data that emphasizes the importance of considering overall cost and adherence information in conjunction with assessment of stroke and bleeding risk to inform the overall risk-benefit of NOAC therapy. Adherence to NOACs proportionally increased with a higher risk of stroke and bleeding and level of comorbidity.

Study results showed that better adherence to NOACs leads to substantial overall cost savings $(-\$ 3,867)$ over a follow-up period of 12 months, and the cost burden significantly increased with a higher risk of bleeding and a higher level of comorbidity.

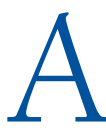
trial fibrillation (AF) is a common condition that causes cardiac rhythm disturbance because of abnormal impulse formation, which can lead to numerous heart-related complications. ${ }^{1} \mathrm{AF}$ is one of the key risk factors for ischemic stroke, increasing the risk up to 5-fold. ${ }^{2}$ In 2010, the prevalence of AF in the United States was 2.7 million and is expected to grow to 5.6 million by $2050 .^{3}$ AF accounts for a substantial portion of the U.S. health care burden, with more than 467,000 hospitalizations annually and more than 99,000 deaths per year. AF is also responsible for adding $\$ 26$ billion to U.S. health care spending annually, which is mostly driven by inpatient and outpatient costs. ${ }^{1}$ Adherence to anticoagulant therapy and accurate assessment of stroke and bleeding risk are vital for reducing the related health care burden and ensuring treatment success.

Novel oral anticoagulants (NOACs) are a new class of drugs that offer benefits such as quick time-to-peak effects, fewer drug-drug and dietary interactions, fixed dosing regimens, and minimal international normalized ratio (INR) monitoring. Overall, NOACs have shown better or similar efficacy in the reduction of stroke risk and noninferior risk of major 
bleeding versus warfarin in the clinical trials. NOACs have also been prescribed for treatment of deep vein thrombosis and pulmonary embolism. ${ }^{4,5}$

Adherence to long-term medication therapy is crucial for achieving efficacy and reducing cost and hospitalizations. Long-term treatment with NOACs is recommended for patients with AF for stroke prevention ${ }^{1}$; however, adherence is suboptimal, with approximately half of patients being adherent to oral anticoagulation therapy. ${ }^{6-9}$

A study comparing the costs of rivaroxaban and warfarin using a Humana claims database reported that health care costs were comparable. The mean total costs for rivaroxaban was slightly lower compared with warfarin $(\$ 17,590$ vs. $\$ 18,676)$, but higher pharmacy cost for rivaroxaban was offset by lower hospitalization cost. ${ }^{10}$ Similar cost comparison results were found between dabigatran and warfarin, using HealthCore data. ${ }^{11}$ Overall, based on recently published literature, NOACs tend to demonstrate better or comparable economic outcomes than warfarin, but no data exist to highlight the effect of better adherence to NOACs on cost savings.

With inadequate real-world evidence, the challenge for drug reimbursement policy decision makers is to consider whether the high drug costs and copays for NOACs are offset by lower overall medical costs, better adherence, and quality of life. A comprehensive evaluation of overall cost and its components (drug and medical costs) in patients treated with NOACs across different levels of adherence, comorbidities, and stroke and bleeding risk is not yet available. Furthermore, the association of improved adherence NOACs and consequent cost savings has not yet been examined in real-world studies. This analysis addressed these gaps in the current literature.

\section{Methods}

\section{Study Design and Cohort}

A retrospective cohort study was conducted with deidentified medical and pharmacy claims data from January 1, 2010, to December 31, 2012, using a nationwide commercial U.S. managed care health plan affiliated with Optum Clinformatics Data Mart (Optum Insight, Eden Prairie, MN). Adult patients were included who were aged $\geq 18$ years with at least $1 \mathrm{AF}$ or atrial-flutter diagnosis claim identified using the medical file (inpatient or outpatient) with an International Classification of Diseases, Ninth Revision, Clinical Modification (ICD-9-CM) code of 427.31/427.32 during the pre-index period. Patients with at least 2 pharmacy claims of dabigatran or rivaroxaban (NOACs) were identified based on National Drug Code numbers. Patients with at least 6 months of pre-index and 12 months of post-index continuous eligibility with a permissible gap of 45 days were included in the cohort. Patients with hyperthyroidism (ICD-9-CM code 242.9) were excluded from the cohort, since hyperthyroidism may be the probable cause of AF. The $\mathrm{CHA}_{2} \mathrm{DS}_{2} \mathrm{VASc}$ score was used to quantify the stroke risk and was scored from 0-9 points based on risk factors for stroke that included congestive heart failure, hypertension, age 65-75 years, diabetes, peripheral vascular disease, female gender (constituting 1 point each), and previous occurrence of transient ischemic attack or stroke and age $\geq 75$ years scored 2 points. ${ }^{12-14}$ Patients with $\mathrm{CHA}_{2} \mathrm{DS}_{2}$ VASc scores $\geq 1$ in the preindex period were included in the cohort. ${ }^{13,14}$

The index date was defined as the date of the first prescription fill of NOAC medication during the measurement period. Patients with concomitant use of warfarin and NOACs during the post-index period were excluded. To avoid exclusion of the few patients who used warfarin in the pre-index period before treatment with NOACs, NOAC users with pre-index warfarin use were included based on the definition of "warfarin-naive" that was applied in the Randomized Evaluation of Long-Term Anticoagulation Therapy (RELY) trials. Patients were considered to be warfarin-naive if they had not used warfarin during the 2 months before initiation of a NOAC. ${ }^{15}$ Data from the RELY trials showed no heterogeneity between patients who had previous warfarin use and those with no previous warfarin therapy. We used this criterion to ensure that we captured all NOAC users and to avoid potential channeling bias regarding previous warfarin therapy for assessment of outcomes. Thus, the index date of warfarin-naive users was based on the first prescription fill of NOACs. Patient outcomes were assessed over a post-index follow-up period of 12 months. The assessment period included index date to 3 months, 6 months, and 12 months.

\section{Measurement of Medication Adherence}

Proportion of days covered (PDC) was the preferred method to measure adherence, where the numerator was defined as number of days covered by NOAC drugs as a class (using first fill date and days of supply) and the denominator was the days between first fill and end of the study, disenrollment, or death, whichever occurred first. PDC also helped to adjust early refills. ${ }^{16}$ Overall adherence to NOAC use was calculated using PDC from the index date to the end of the assessment period $(3,6$, and 12 months). Patients with a PDC $\geq 80 \%$ were classified as adherent. ${ }^{6,17}$ Adherence was also calculated for an assessment period of 3,6 , and 12 months using the following 3 severity groups:

1. Stroke risk based on $\mathrm{CHA}_{2} \mathrm{DS}_{2}$ VASc score. Risk was categorized as low risk $\left(\mathrm{CHA}_{2} \mathrm{DS}_{2}\right.$ VASc score of 1$)$, moderate risk $\left(\mathrm{CHA}_{2} \mathrm{DS}_{2} \mathrm{VASc}\right.$ score of $\left.2-3\right)$, and high risk $\left(\mathrm{CHA}_{2} \mathrm{DS}_{2} \mathrm{VASc}\right.$ score of 4+).

2. Bleeding risk based on HAS-BLED score calculated using hypertension, renal disease, liver disease, antiplatelet or nonsteroidal anti-inflammatory drug use, stroke history, previous bleeding, age $\geq 65$ years, labile INR (therapeutic time in range $<60 \%$ ), and alcohol or drug use constituting 


\section{Real-World Health Care Costs Based on Medication Adherence and Risk of Stroke and Bleeding in Patients Treated with Novel Anticoagulant Therapy}

\section{FIGURE 1 Cohort Selection Based on Inclusion and Exclusion Criteria}

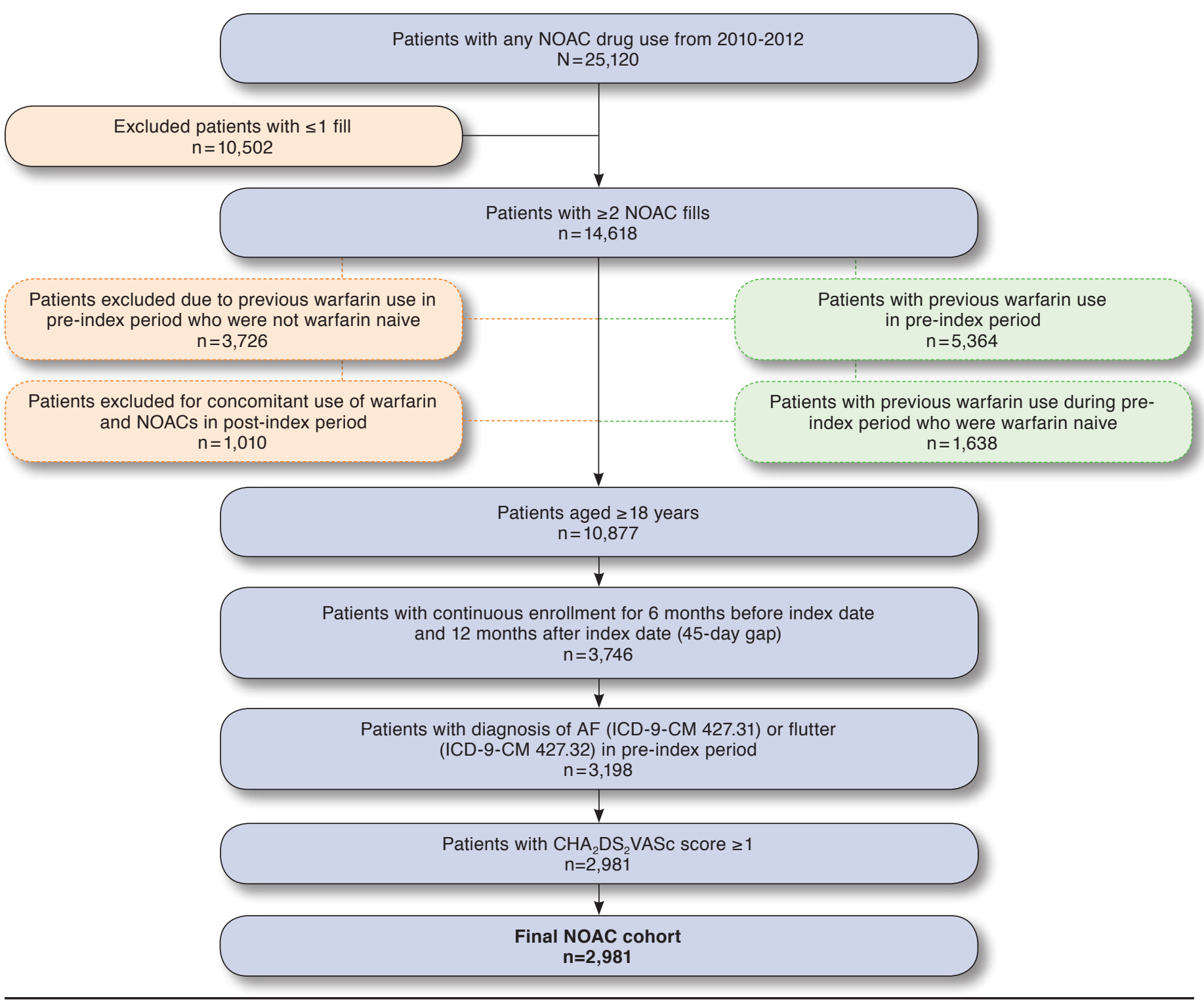

AF = atrial fibrillation; ICD-9-CM=International Classification of Diseases, Ninth Revision, Clinical Modification; NOAC=novel oral anticoagulant.

1 point each. ${ }^{14}$ Risk was categorized as low risk (HAS-BLED score of 0-1), moderate risk (HAS-BLED score of 2), and high risk (HAS-BLED score of 3+). Because data on labile INR values were unavailable, all patients were scored 0 in this category.

3. Comorbidity using Charlson Comorbidity Index (CCI) scores based on the adapted version by Deyo et al. (1992) for administrative claims data. ${ }^{14,18,19} \mathrm{CCI}$ scores were subgrouped as $\mathrm{CCI}=0, \mathrm{CCI}=1-2$, and $\mathrm{CCI}=3+$ comorbid disease conditions. ${ }^{18}$

\section{Cost}

The per-patient post-index cost for a follow-up of 12 months was calculated. The medical cost was defined as the total amount paid for inpatient and outpatient medical services plus inpatient and outpatient professional fees and copays. The total health care cost was the sum of all medical and drug costs using the amount paid by the patient through prescription fills (pharmacy claims and copays). The cost measures were expressed as average per-patient annual all-cause costs. The 


\section{Real-World Health Care Costs Based on Medication Adherence and Risk of Stroke and Bleeding in Patients Treated with Novel Anticoagulant Therapy}

TABLE 1 Demographic Characteristics of Overall Sample and Cohorts Based on Adherence

\begin{tabular}{|c|c|c|c|c|c|}
\hline Variable Description & $\begin{array}{c}\text { Statistic Response } \\
\text { Category }\end{array}$ & $\begin{array}{c}\text { Total } \\
(\mathrm{N}=2,981)\end{array}$ & $\begin{array}{l}\text { Adherent }^{\mathrm{a}} \\
(\mathrm{n}=1,621)\end{array}$ & $\begin{array}{l}\text { Nonadherent } \\
(\mathrm{n}=1,360)\end{array}$ & $\begin{array}{c}P \text { Value } \\
\text { (Chi-square test) }\end{array}$ \\
\hline \multirow[t]{4}{*}{ Age at index } & Number & 2,981 & 1,621 & 1,360 & 0.0009 \\
\hline & Mean (SD) & $64.39 \quad(10.72)$ & $66.53 \quad(10.05)$ & $61.84 \quad(10.95)$ & \\
\hline & Median (IQR) & $63 \quad(58-72)$ & $65 \quad(60-74)$ & $61 \quad(55-68)$ & \\
\hline & Range & $26-86$ & $34-86$ & $26-86$ & \\
\hline \multirow[t]{2}{*}{ Gender, n (\%) } & Female & $898 \quad(30.13)$ & $501 \quad(30.91)$ & $397 \quad(29.19)$ & 0.3039 \\
\hline & Male & $2,082 \quad(69.87)$ & $1,119 \quad(69.03)$ & $963 \quad(70.81)$ & \\
\hline \multirow[t]{5}{*}{ Insurance type, n (\%) } & EPO & $349 \quad(11.71)$ & $172 \quad(10.61)$ & $177 \quad(13.01)$ & $<0.0001$ \\
\hline & $\mathrm{HMO}$ & $190 \quad(6.37)$ & $91 \quad(5.61)$ & $99 \quad(7.28)$ & \\
\hline & IND & $452 \quad(15.16)$ & $(19.31)$ & $139 \quad(10.22)$ & \\
\hline & POS & $1,867 \quad(62.63)$ & $967 \quad(59.65)$ & $900 \quad(66.18)$ & \\
\hline & $\mathrm{PPO}$ & $122 \quad(4.09)$ & $77 \quad(4.75)$ & $45 \quad(3.31)$ & \\
\hline \multirow[t]{4}{*}{ Region, n (\%) } & Midwest & $636 \quad(21.34)$ & $370 \quad(22.83)$ & $266 \quad(19.56)$ & $<0.0001$ \\
\hline & Northeast & $306 \quad(10.27)$ & $164 \quad(10.12)$ & $142 \quad(10.44)$ & \\
\hline & South & $1,525 \quad(51.17)$ & $768 \quad(47.38)$ & $757 \quad(55.66)$ & \\
\hline & West & $513 \quad(17.21)$ & $(19.68)$ & $194 \quad(14.26)$ & \\
\hline \multirow{3}{*}{$\begin{array}{l}\text { Stroke risk } \\
\left(\mathrm{CHA}_{2} \mathrm{DS}_{2} \text { VASc score }\right)^{\mathrm{b}} \\
\mathrm{n}(\%)\end{array}$} & Low risk & $575 \quad(19.29)$ & $(14.62)$ & $338 \quad(24.85)$ & $<0.0001$ \\
\hline & Moderate risk & $1,336 \quad(44.82)$ & $692 \quad(42.69)$ & $644 \quad(47.35)$ & \\
\hline & High risk & $1,070 \quad(35.89)$ & $(42.69)$ & $378 \quad(27.79)$ & \\
\hline \multirow[t]{3}{*}{ CCI category, n (\%) } & CCI score 0 & $602 \quad(20.19)$ & $(17.52)$ & $318 \quad(23.38)$ & 0.0002 \\
\hline & CCI score 1-2 & $1,403 \quad(47.06)$ & $773 \quad(47.69)$ & $630 \quad(46.32)$ & \\
\hline & CCI score $3+$ & $976 \quad(32.74)$ & $(34.79)$ & $412 \quad(30.29)$ & \\
\hline \multirow[t]{2}{*}{ Statin use, n (\%) } & No & $1,512 \quad(50.72)$ & $(45.34)$ & $777 \quad(57.13)$ & $<0.0001$ \\
\hline & Yes & $1,469 \quad(49.28)$ & $886 \quad(54.66)$ & $583 \quad(42.87)$ & \\
\hline \multirow[t]{2}{*}{ ACE or ARB use, n (\%) } & No & $1,551 \quad(52.03)$ & $(48.43)$ & $766 \quad(56.32)$ & $<0.0001$ \\
\hline & Yes & $1,430 \quad(47.97)$ & $836 \quad(51.57)$ & $594 \quad(43.68)$ & \\
\hline \multirow[t]{2}{*}{ Beta blocker use, n (\%) } & No & $2,065 \quad(69.27)$ & $(68.23)$ & $959 \quad(70.51)$ & 0.1780 \\
\hline & Yes & $916 \quad(30.73)$ & $515 \quad(31.77)$ & $401 \quad(29.49)$ & \\
\hline \multirow{3}{*}{$\begin{array}{l}\text { Bleeding risk (HAS-BLED) } \\
\text { n (\%) }\end{array}$} & Low risk & $1,084 \quad(36.36)$ & $496 \quad(30.60)$ & $588 \quad(43.24)$ & $<0.0001$ \\
\hline & Moderate risk & $1,028 \quad(34.49)$ & $605 \quad(37.32)$ & $423 \quad(31.10)$ & \\
\hline & High risk & $869 \quad(29.15)$ & $520 \quad(32.08)$ & $349 \quad(25.66)$ & \\
\hline
\end{tabular}

aAdherence was based on 12-month follow-up, and the adherence cohort was defined based on PDC $\geq 80 \%$.

bStroke risk was categorized as low risk $\left(\mathrm{CHA}_{2} \mathrm{DS} \mathrm{S}_{2} \mathrm{VASC}\right.$ score of 1), moderate risk $\left(\mathrm{CHA}_{2} \mathrm{DS}_{2} \mathrm{VASC}\right.$ score of 2-3), and high risk $\left(\mathrm{CHA}_{2} \mathrm{DS} \mathrm{S}_{2} \mathrm{VASC}\right.$ score of 4+).

cBleeding risk was classified as low risk (HAS-BLED score of 0-1), moderate risk (HAS-BLED score of 2), and high risk (HAS-BLED score of 3+).

$A C E=$ angiotensin converting enzyme inhibitor; $A R B=$ angiotensin receptor blocker; $C C I=C h a r l s o n$ Comorbidity Index; $E P O=$ exclusive provider organization;

$H M O=$ health maintenance organization; IND = indemnity insurance; $I Q R=$ interquartile range; $P O S=$ point of service; $P P O=$ preferred provider organization;

$S D=$ standard deviation

cost incurred for the years 2010-2012 was adjusted for inflation to 2016 U.S. dollars based on the Consumer Price Index.

\section{Multivariate Analysis}

A logistic regression model was used to examine the predictors of medication adherence based on PDC $\geq 80 \%$ over 12 months post-index (dependent variable, 1/0). Age, region, insurance plan type, gender at index date of NOAC use, pre-index CCI, stroke risk based on $\mathrm{CHA}_{2} \mathrm{DS}_{2}$ VASc score, bleeding risk based on HAS-BLED score, and cardiac medication use that included statin use, angiotensin-converting enzyme inhibitor or angiotensin receptor blockers (ACE-ARB) use, and beta blocker use were used as covariates and included in the full model.
Generalized linear models with gamma distribution and log link were also used to obtain and compare the adjusted annual costs between adherent and nonadherent patients, defined based on $\mathrm{PDC} \geq 80 \%$. The gamma distribution was preferred, since we expected a skewed distribution of the cost, and the gamma model is an acceptable approach to handle cost data. ${ }^{20}$ Age, CCI scores, stroke risk based on $\mathrm{CHA}_{2} \mathrm{DS}_{2}$ VASc scores, region, insurance type, gender, bleeding risk based on HASBLED scores, and cardiac medication use including statin, ACE-ARB, and beta blocker use were used as covariates. The total annual cost, along with its subcomponents (drug cost and medical cost) were also compared between the patients stratified based on CCI score and stroke and bleeding risk at 


\section{Real-World Health Care Costs Based on Medication Adherence and Risk of Stroke and Bleeding in Patients Treated with Novel Anticoagulant Therapy}

TABLE 2 Adherence to NOAC Therapy over 12-Month Follow-up for Stroke and Bleeding Risk (N=2,981)

\begin{tabular}{|c|c|c|c|c|}
\hline \multirow{3}{*}{$\begin{array}{l}\text { Outcome Cohort } \\
\text { Overall adherence }\end{array}$} & \multirow{3}{*}{$\begin{array}{c}\text { Overall } \\
\mathbf{N} \\
2,981\end{array}$} & \multicolumn{3}{|c|}{ Patient Adherence PDC $\geq 80 \%$} \\
\hline & & $\begin{array}{l}\text { 3-Month } \\
\text { Follow-up } \\
\text { n (\%) }\end{array}$ & $\begin{array}{l}\text { 6-Month } \\
\text { Follow-up } \\
\mathrm{n}(\%)\end{array}$ & $\begin{array}{l}\text { 12-Month } \\
\text { Follow-up } \\
\text { n (\%) }\end{array}$ \\
\hline & & $2,144 \quad(71.92)$ & $1,935 \quad(64.91)$ & $1,621 \quad(54.38)$ \\
\hline \multicolumn{5}{|c|}{ Adherence by stroke risk ${ }^{a}$} \\
\hline Low risk & 575 & $344 \quad(59.83)$ & $300 \quad(52.17)$ & $237 \quad(41.22)$ \\
\hline Moderate risk & 1,336 & $946 \quad(70.81)$ & $835 \quad(62.50)$ & $692 \quad(51.80)$ \\
\hline High risk & 1,070 & $854 \quad(79.81)$ & $800 \quad(74.77)$ & $692 \quad(64.67)$ \\
\hline Overall $P$ value & & $<0.0001$ & $<0.0001$ & $<0.0001$ \\
\hline \multicolumn{5}{|c|}{ Adherence by bleeding risk ${ }^{b}$} \\
\hline Low risk & 1,084 & $711 \quad(65.59)$ & $623 \quad(57.47)$ & $496 \quad(45.76)$ \\
\hline Moderate risk & 1,028 & $778 \quad(75.68)$ & $708 \quad(68.87)$ & $605 \quad(58.85)$ \\
\hline High-risk & 869 & $655 \quad(75.37)$ & $604 \quad(69.51)$ & $520 \quad(59.84)$ \\
\hline Overall $P$ value & & $<0.0001$ & $<0.0001$ & $<0.0001$ \\
\hline \multicolumn{5}{|l|}{ Adherence by CCIc } \\
\hline Low risk & 602 & $408 \quad(67.77)$ & $355 \quad(58.97)$ & $(47.18)$ \\
\hline Moderate risk & 1,403 & $1,011 \quad(72.06)$ & $917 \quad(65.36)$ & $773 \quad(55.10)$ \\
\hline High risk & 976 & $725 \quad(74.28)$ & $663 \quad(67.93)$ & $564 \quad(57.79)$ \\
\hline Overall $P$ value & & 0.0199 & 0.0013 & 0.0002 \\
\hline $\begin{array}{l}\text { aStroke risk was cates } \\
\text { bBleeding risk was cla } \\
{ }^{c} \text { CCI scores were subs } \\
\text { CCI = Charlson Como }\end{array}$ & $\begin{array}{l}\mathrm{CCI}=3+ \\
\text { oral antice }\end{array}$ & $\begin{array}{l}\text { risk }\left(\mathrm{CHA}_{2} \mathrm{DS}_{2} \mathrm{VA}\right. \\
\text { sk ( } \mathrm{HAS}-\mathrm{BLED} \text { sc } \\
\text { ins. } \\
\text { roportion of days }\end{array}$ & $\begin{array}{l}\text { and high risk (C } \\
\text { gh risk (HAS-BI }\end{array}$ & re of $4+$ ). \\
\hline
\end{tabular}

a significance level of $P \leq 0.05$. Data were analyzed using SAS Enterprise Guide version 7.1 (SAS Institute, Cary, NC).

\section{Results}

\section{Cohort Selection}

Of the 25,120 NOAC users identified during the study period, 14,618 had 2 or more prescription fills. Of these patients, 5,364 patients had used warfarin in the pre-index period, and, 3,726 patients who were not warfarin naive had been excluded, leaving 1,638 patients had been included as NOAC users because they fit the definition of warfarin naive. Because of concomitant use of warfarin and NOACs in the post-index period, 1,010 patients had also been excluded. Based on the other inclusion criteria (diagnosis of AF in the pre-index period, aged $\geq 18$ years, continuous enrollment for 6-month pre-index and 12 -month post-index, and a $\mathrm{CHA}_{2} \mathrm{DS}_{2} \mathrm{VASc}$ score $\geq 1$ ), 2,891 NOAC patients formed the final cohort (Figure 1).

\section{Overall Baseline Characteristics Across Cohorts}

The mean age of the cohort was 64 years, with more men (70\%) than women. Most of the patients were either from the South or the Midwest (68\%). The proportion of patients with moderate and high risk of stroke was higher (high 36\% vs. moderate $45 \%$ vs. low 19\%) compared with low-risk patients. Based on CCI scores, most (80\%) of the patients had at least 1 comorbid condition. Patients were well distributed across bleeding risk based on the HAS-BLED scores. More than 45\% of the cohort used statins and ACE-ARB class of cardiovascular drugs (Table 1).

\section{Baseline Characteristics Among Adherent and Nonadherent NOAC Patients}

Based on baseline characteristics, age, stroke risk, type of insurance, statin use, bleeding risk, CCI, and region were significantly $(P \leq 0.05)$ different across the adherent and nonadherent patient groups. The mean age of patients classified as adherent was slightly higher (approximately 67 years [standard deviation $\{\mathrm{SD}\}=10]$ ) compared with the nonadherent group (approximately 62 years $[S D=11]$ ). Gender and beta blocker use was consistent between the adherence-based cohorts. The proportion of patients with moderate to high risk of stroke $\left(\mathrm{CHA}_{2} \mathrm{DS}_{2} \mathrm{VASc}\right.$ score $\left.\geq 4\right)$ was substantially higher among adherent versus nonadherent patients (43\% vs. $28 \%$; Table 1).

\section{Adherence Measured by PDC}

Adherence was measured during follow-up periods of 3, 6, and 12 months. In the overall sample, the proportion of adherent patients ( $\mathrm{PDC} \geq 80 \%$ ) declined during the period from 3, 6 , and 12 months (72\% for 3-month follow-up, 65\% for 6-month follow-up, and $54 \%$ for 12 -month follow-up). This trend was evident across all subgroups based on $\mathrm{CCI}, \mathrm{CHA}_{2} \mathrm{DS}_{2} \mathrm{VASc}$, and HAS-BLED scores (Table 2).

Adherence increased with a higher risk of stroke. Based on the 12-month follow-up, the proportion of patients adherent 


\section{Real-World Health Care Costs Based on Medication Adherence and Risk of Stroke and Bleeding in Patients Treated with Novel Anticoagulant Therapy}

\begin{tabular}{|c|c|c|c|c|c|c|c|}
\hline \multirow{2}{*}{$\begin{array}{l}\text { Outcome } \\
\text { Age }\end{array}$} & \multirow[t]{2}{*}{ Reference } & \multirow{2}{*}{$\begin{array}{c}\text { Effect } \\
-\end{array}$} & \multirow{2}{*}{$\begin{array}{c}\text { Odds Ratio } \\
1.035\end{array}$} & \multicolumn{2}{|c|}{$95 \% \mathrm{CI}$} & \multirow{2}{*}{$\begin{array}{c}P \text { Value } \\
<0.0001\end{array}$} & \multirow{2}{*}{$\begin{array}{l}\text { Overall } \\
P \text { Value } \\
<0.0001\end{array}$} \\
\hline & & & & 1.024 & 1.046 & & \\
\hline Gender & Male & Female vs. male & 0.907 & 0.762 & 1.079 & 0.2702 & 0.2702 \\
\hline \multirow[t]{4}{*}{ Insurance type } & \multirow[t]{4}{*}{$\mathrm{HMO}$} & EPO vs. HMO & 1.240 & 0.860 & 1.789 & 0.9702 & 0.5744 \\
\hline & & IND vs. HMO & 1.395 & 0.952 & 2.045 & 0.9723 & \\
\hline & & POS vs. HMO & 1.235 & 0.906 & 1.684 & 0.9702 & \\
\hline & & PPO vs. HMO & 1.520 & 0.932 & 2.479 & 0.9738 & \\
\hline \multirow[t]{3}{*}{ Region } & \multirow[t]{3}{*}{ Midwest } & Northeast vs. Midwest & 0.738 & 0.553 & 0.985 & 0.1088 & 0.0072 \\
\hline & & South vs. Midwest & 0.755 & 0.619 & 0.920 & 0.0301 & \\
\hline & & West vs. Midwest & 0.990 & 0.771 & 1.272 & 0.0891 & \\
\hline \multirow[t]{2}{*}{ Stroke riska } & \multirow[t]{2}{*}{ Low risk } & High risk vs. low risk & 1.556 & 1.144 & 2.117 & 0.0050 & 0.0173 \\
\hline & & Moderate risk vs. low risk & 1.209 & 0.967 & 1.512 & 0.7013 & \\
\hline \multirow[t]{2}{*}{$\mathrm{CCI}^{\mathrm{b}}$} & \multirow[t]{2}{*}{$\mathrm{CCI}=0$} & CCI score $1-2$ vs. CCI score 0 & 1.249 & 1.011 & 1.542 & 0.0131 & 0.0453 \\
\hline & & CCI score $3+$ vs. CCI score 0 & 1.051 & 0.811 & 1.362 & 0.5536 & \\
\hline Statin use & No use & Yes vs. no use & 1.305 & 1.114 & 1.530 & 0.0010 & 0.0010 \\
\hline ACE-ARB use & No use & Yes vs. no use & 1.187 & 1.015 & 1.389 & 0.0320 & 0.0320 \\
\hline Beta blocker & No use & Yes vs. no use & 1.032 & 0.874 & 1.218 & 0.7109 & 0.7109 \\
\hline \multirow[t]{2}{*}{ Bleeding risk ${ }^{c}$} & \multirow[t]{2}{*}{ Low risk } & High.risk vs. low risk & 0.760 & 0.584 & 0.990 & 0.0067 & 0.0100 \\
\hline & & Moderate risk vs. low risk & 1.069 & 0.877 & 1.303 & 0.0139 & \\
\hline \multicolumn{8}{|c|}{ 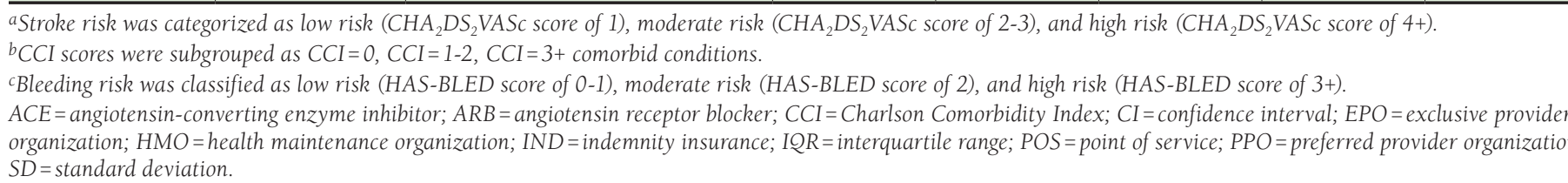 } \\
\hline
\end{tabular}

to NOACs in the low-risk and moderate-risk groups were $41 \%$ and $52 \%$, respectively, and were significantly $(P<0.001)$ lower compared with the proportion of adherent patients (65\%) in the high-risk group. Similar results were observed during the follow-up periods of 3 and 6 months. Higher adherence among higher risk groups was evident in the subgroups based on bleeding risk and CCI scores over the 3 follow-up periods (3, 6, and 12 months; Table 2).

\section{Predictors of Medication Adherence}

Based on the logistic regression model, age, region, CCI scores, stroke risk, statin and ACE-ARB use, and bleeding risk were significant predictors of medication adherence to NOACs. Adherence rate was higher with increasing age (odds ratio $[\mathrm{OR}]=1.04,95 \%$ confidence interval $[\mathrm{CI}]=1.02-1.05$ ), and patients in the Midwest had higher adherence compared with the other regions. Patients with higher stroke risk were 1.56 times (95\% CI=1.14-2.12) more likely to be adherent compared with low-risk patients. Similarly, patients with CCI scores of $1-2$ had higher odds (OR $=1.25,95 \% \mathrm{CI}=1.01-1.54)$ of being adherent than those with CCI scores of 0 . Patients using statins and ACE-ARBs tended to be more adherent (OR for statins $=1.31,95 \% \mathrm{CI}=1.11-1.53$ and OR for ACE-ARBs $=1.19$, 95\% CI $=1.02-1.39)$ to NOAC therapy compared with patients who were not taking these cardiovascular medications. Finally, patients with a moderate risk of bleeding were 1.07 times (95\% $\mathrm{CI}=0.88$-1.30) more likely to be adherent compared with lowrisk patients (Table 3).

\section{Comparison of Annual All-Cause Health Care Costs}

As expected, the annual adjusted per-patient drug cost for adherent users was significantly higher compared with nonadherent users ( $\$ 5,595$ vs. $\$ 2,233 ; P \leq 0.001$ ). However, the annual adjusted per-patient medical cost that included all inpatient and outpatient costs was significantly lower for adherent users compared with nonadherent users ( $\$ 23,544$ vs. $\$ 30,485$; $P \leq 0.001)$. The total annual adjusted per-patient cost for adherent users was also significantly lower than nonadherent users $(\$ 29,742$ vs. $\$ 33,609 ; P=0.005)$. Overall, the high drug cost among adherent users was offset by lower inpatient and outpatient costs (medical costs) compared with nonadherent users (Table 4).

\section{Comparison of Adjusted Costs by Stroke Risk}

Most of the patients had moderate to high risk of stroke based on the $\mathrm{CHA}_{2} \mathrm{DS}_{2}$ VASc score (low risk $19.29 \%$, moderate risk $44.82 \%$, and high risk $35.89 \%$ ). The annual per-patient drug cost was slightly lower in patients with low risk of stroke compared with patients with moderate and high risk (low risk $\$ 3,347$ vs. moderate risk $\$ 3,664$ vs. high risk $\$ 3,602$ ). The 


\section{Real-World Health Care Costs Based on Medication Adherence and Risk of Stroke and Bleeding in Patients Treated with Novel Anticoagulant Therapy}

\begin{tabular}{|c|c|c|c|c|}
\hline Outcome Cohort & Overall N & $\begin{array}{l}\text { Average Adjusted Annual } \\
\text { Pharmacy Cost, \$ }(95 \% \text { CI })^{\mathrm{a}}\end{array}$ & $\begin{array}{l}\text { Average Adjusted Annual } \\
\text { Medical Cost, \$ }(95 \% \mathrm{CI})^{\mathrm{a}}\end{array}$ & $\begin{array}{c}\text { Average Adjusted Annual } \\
\text { Total Cost, \$ }(95 \% \mathrm{CI})^{\mathrm{a}}\end{array}$ \\
\hline \multicolumn{5}{|l|}{ Adherence } \\
\hline Nonadherent & 1,360 & $(1,898-2,628)$ & $30,485 \quad(21,348-43,535)$ & $33,609 \quad(24,938-45,297)$ \\
\hline Adherent & 1,621 & $5,595 \quad(4,758-6,750)$ & $23,544(16,506-33,583)$ & $29,742 \quad(22,091-40,043)$ \\
\hline Overall $P$ value & & $<0.0001$ & $<0.0001$ & 0.0005 \\
\hline \multicolumn{5}{|c|}{ Stroke risk $\left(\mathrm{CHA}_{2} \mathrm{DS}_{2} \mathrm{VASc}\right)^{\mathrm{b}}$} \\
\hline Low risk & 575 & $(2,830-3,958)$ & $33,007 \quad(22,849-47,681)$ & $37,972 \quad(27,906-51,668)$ \\
\hline Moderate risk & 1,336 & $3,664 \quad(3,113-4,312)$ & $27,271 \quad(19,081-38,976)$ & $32,036 \quad(23,756-43,201)$ \\
\hline High risk & 1,070 & $3,602 \quad(3,055-4,247)$ & $21,362 \quad(14,901-36,626)$ & $25,981 \quad(19,214-35,242)$ \\
\hline Overall $P$ value & & 0.0049 & $<0.0001$ & $<0.0001$ \\
\hline \multicolumn{5}{|c|}{ Bleeding risk (HAS-BLED)c } \\
\hline Low risk & 1,084 & $(3,053-4,236)$ & $22,516 \quad(15,744-32,201)$ & $27,189 \quad(20,149-36,689)$ \\
\hline Moderate risk & 1,028 & $(2,978-4,132)$ & $24,916 \quad(17,411-35,656)$ & $29,641 \quad(21,956-40,018)$ \\
\hline High risk & 869 & $3,501 \quad(2,966-4,132)$ & $34,276 \quad(23,823-49,315)$ & $39,216 \quad(28,919-53,179)$ \\
\hline Overall $P$ value & & 0.5752 & $<0.0001$ & $<0.0001$ \\
\hline \multicolumn{5}{|l|}{$\mathrm{CCI}^{\mathrm{d}}$} \\
\hline Low risk & 602 & $(2,911-4,059)$ & $18,482 \quad(12,828-26,609)$ & $22,912 \quad(16,886-31,089)$ \\
\hline Moderate risk & 1,403 & $(3,037-4,207)$ & $25,149 \quad(17,603-35,929)$ & $29,719 \quad(22,043-40,066)$ \\
\hline High risk & 976 & $3,595 \quad(3,052-4,234)$ & $41,369 \quad(28,910-59,199)$ & $46,416 \quad(34,384-62,657)$ \\
\hline Overall $P$ value & & 0.2893 & $<0.0001$ & $<0.0001$ \\
\hline \multicolumn{5}{|c|}{ 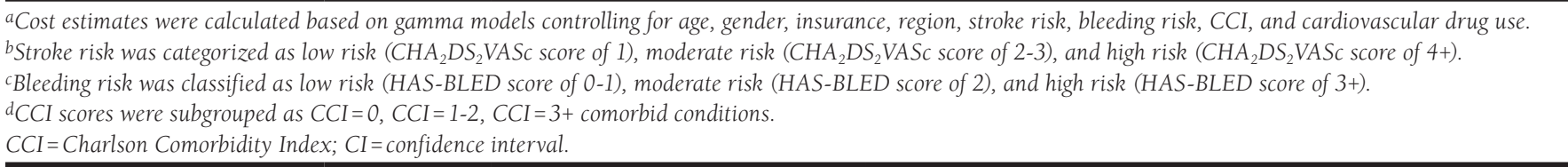 } \\
\hline
\end{tabular}

annual per-patient medical cost for NOAC users with high risk of stroke was lower than the cost of patients with low risk of stroke. A similar trend was evident for the total cost in which the cost incurred by high-risk patients was lower than the cost incurred by low-risk patients (Table 4).

\section{Comparison of Adjusted Costs by Bleeding Risk}

Patients were well distributed by bleeding risk based on the HAS-BLED score (low risk 36.36\%, moderate risk 34.49\%, and high risk 29.15\%). The annual adjusted per-patient drug cost did not significantly differ across groups based on the risk of bleeding (low risk $\$ 3,596$ vs. moderate risk $\$ 3,508$ vs. high risk $\$ 3,501$ ). However, the annual adjusted per-patient medical cost increased significantly with an increased bleeding risk (low risk $\$ 22,516$ vs. moderate risk $\$ 24,916$ vs. high risk $\$ 34,276 ; P \leq 0.001)$. High medical cost was the driver for the total annual cost, in which the same trend of higher total cost with increased risk of bleeding was consistent (Table 4).

\section{Comparison of Adjusted Costs by CCI}

Most of the patients had at least 1 comorbidity (79.8\%) based on CCI scores. There were $32.74 \%$ of patients with 3 or more comorbid conditions. Similar to the subgroups based on bleeding risk, the annual adjusted per-patient drug cost did not significantly differ across groups based on CCI scores. However, the total adjusted per-patient cost increased significantly with increase in CCI scores (low risk $\$ 27,189$ vs. moderate risk $\$ 29,641$ vs. high risk $\$ 39,216 ; P \leq 0.001$ ), which was mostly driven by the high medical costs across highly comorbid patients (Table 4)

\section{Discussion}

This study found that the economic burden of NOAC users based on total annual health care cost was substantial $(>\$ 30,000)$. However, better long-term adherence to NOACs directly translated in overall cost savings. The medical (inpatient and outpatient) costs for adherent users were significantly lower $(-\$ 6,941)$ than nonadherent patients, which offset the higher drug cost $(+\$ 3,362)$ among adherent users. The drug cost was comparable between groups categorized based on stroke risk, bleeding risk, and CCI scores. However, total cost was driven mostly by medical cost, which increased proportionally with a higher risk of bleeding and comorbidity. A similar trend was not observed for cost by stroke risk.

We also found that adherence to NOACs in our sample was suboptimal $(<60 \%$ with $\mathrm{PDC} \geq 80 \%)$ and was better among patients with higher comorbidity and a higher risk of stroke and bleeding. The high-risk population benefited from 
frequent physician visits, focused interventions, aggressive efforts by physicians for improving care and adherence to treatment guidelines, better awareness among severe patients, and motivation to feel better, which can potentially lead to better patient-provider interaction and improvement in overall adherence among these patients.

Based on a large database study by Yao et al. (2016), adherence to NOACs at the end of follow-up at 1.1 years was low (47.5\%), which is similar to our estimate of adherence (54\%) at 12 months. ${ }^{6}$ Based on another study using commercial insured patients taking NOACs, the proportion of patients with $\mathrm{PDC} \geq 80 \%$ at 9 months was suboptimal, with $55.0 \%$ for rivaroxaban, $56.8 \%$ for apixaban, and $46.7 \%$ for dabigatran users. ${ }^{21}$ It is noteworthy that advantages of NOAC over warfarin are promising and that NOAC therapy has a potential to improve overall medication adherence, which is evident from recently published studies. ${ }^{22-24}$

In our sample, it was clearly observed that adherence to NOACs was driven by baseline risk of stroke and bleeding and CCI scores. A study by Brown et al. (2016) reported an increase in adherence to NOAC medication from $58 \%$ in patients with $\mathrm{CHA}_{2} \mathrm{DS}_{2}$ VASc scores of 2-3 and $62 \%$ in patients with a higher risk of stroke $\left(\mathrm{CHA}_{2} \mathrm{DS}_{2} \mathrm{VASc}\right.$ score of $\left.4+\right)$ based on the 9-month follow-up. ${ }^{21}$ Another study based on insurance claims data concluded that patients with a higher risk of stroke were more adherent to NOAC medication than patients at lower risk $\left(\mathrm{CHA}_{2} \mathrm{DS}_{2}\right.$ VASc score 0 or $1,30.5 \%$; $\mathrm{CHA}_{2} \mathrm{DS}_{2}$ VASc score 2 or 3, 43.4\%; and $\mathrm{CHA}_{2} \mathrm{DS}_{2}$ VASc score $\left.\geq 4,45.3 \%\right) .{ }^{6}$ Our study differs from these studies because it presents new evidence in the form of better adherence from groups based on a risk of bleeding and comorbidity, which are integral to the selection of optimal anticoagulation treatment.

Our results also highlighted stroke and bleeding risk as a major predictor of medication adherence. Other predictors such as age, region, CCI score, and statin and ACE-ARB use were consistent with the previous studies., ${ }^{925}$ In addition, younger age, male gender, low stroke risk, poverty, higher education, and poor cognitive function have been associated with lower adherence. ${ }^{28} \mathrm{~A}$ recent study based on the Danish National Patient Registry reported an overall 1-year PDC equal to $83.9 \%$ and found that females ( $\mathrm{OR}=1.06)$, patients using cardiovascular drugs, and a $\mathrm{CHA}_{2} \mathrm{DS}_{2} \mathrm{VASc}$ score of $\geq 2(\mathrm{OR}=1.12)$ were major predictors of adherence among dabigatran users. ${ }^{22}$

Our study is the first to compare the real-world cost between adherent and nonadherent NOAC patients. We found a positive association of adherence with cost saving $(-\$ 3,867)$ over a 12-month period that can be attributed to lower health care utilization (e.g., fewer hospital visits and shorter hospital stays).
Our estimates of health care costs (drug, medical, and total) were consistent with the existing literature. Based on data from the U.S. Department of Defense Military Health System, drug costs were higher $(\$ 4,369, P<0.001)$ for dabigatran compared with warfarin, which is similar to our estimated drug costs..$^{29}$ Also, Bancroft et al. (2016) studied dabigatran costs using a managed care data (2009-2012) and reported annual drug costs as $\$ 6,122$, medical costs as $\$ 19,195$, and total costs as $\$ 25,370 .{ }^{30}$ According to another database study by Fonseca et al. (2015), the total cost for patients taking dabigatran and warfarin after propensity score matching was $\$ 14,794$ vs. $\$ 16,826$. $^{31}$

Drug cost was similar across subgroups based on CCI scores and stroke and bleeding risks. Medical and total costs were higher for patients with higher comorbidities and increased risk of bleeding. Conversely, we found that medical and total costs were higher for patients with a low risk of stroke compared with high-risk patients. Better adherence in the high-risk group might lead to lower incidence of stroke and its related lower cost burden. In addition, complications related to low-risk patients might be aggressively treated, leading to higher medical (inpatient and outpatient) costs. Cost and adherence information by stroke and bleeding risks and CCI scores can serve as crucial parameters informing the risk-benefit of treatment.

One of the strengths of this study was the use of a nationwide database with a large sample size. In addition, HASBLED was preferred because of its widespread use and better discrimination and prediction of bleeding risk compared with HEMORR ${ }_{2}$ HAGES and ATRIA. ${ }^{32,33}$ An inclusion criteria of at least 2 prescription refills ensured the exclusion of possible intermittent users (i.e., multiple periods of use but none exceeding 3 months) or patients who might use NOACs as concomitant therapy or for other indications. Furthermore, 2 prescription refills enabled calculation of adherence using PDC.

\section{Limitations}

A few limitations need to be considered when interpreting our results. Although PDC has been widely used and accepted, it is an indirect method of assessing adherence because it is based on insurance claims (prescription fills). It should also be acknowledged that Optum Clinformatics Data Mart is a nationwide database, but the majority of the study patients were from the southern and midwestern regions. This database also underrepresents the older Medicare population.

As with all studies using claims databases, our data lacked information on race and ethnicity, long-term disease history, reasons for therapy discontinuation, and clinical variables such as INR values and body mass index. However, clinical 
markers of severity, such as $\mathrm{CHA}_{2} \mathrm{DS}_{2}$ VASc, HAS-BLED, and $\mathrm{CCI}$, reflected risk due to hypertension, previous cardiovascular disease, diabetes, and other comorbidities. Aspirin use was not comprehensively captured in the claims database because of its availability as an over-the-counter drug; however, since all patients were prescribed NOACs, the differential use of aspirin might be unlikely among patients with a $\mathrm{CHA}_{2} \mathrm{DS}_{2}$ VASc score $>1$, who are recommended anticoagulant therapy based on guidelines from the American College of Cardiology and the American Heart Association.

It is also important to acknowledge the differences in mechanism of action and costs between dabigatran and rivaroxaban. A higher proportion of our sample was prescribed dabigatran, since it was approved earlier. The newer drugs apixaban and edoxaban, which came into the market after 2012, were not included in our analysis. Since the measurement of PDC and cost was in the same period, inferences suggest a crosssectional association instead of a sequential (longitudinal) causal relationship. This is a question that can be explored in future studies.

Despite these limitations, this study provides new evidence that emphasizes the importance of considering overall cost and adherence information in conjunction with the assessment of stroke and bleeding risk to inform the optimal choice of anticoagulant therapy and ascertain the overall risk-benefit of NOAC therapy.

\section{Conclusions}

Adherence to NOAC therapy over a follow-up period of 12 months was suboptimal (54\%) and increased with a higher level of comorbidities and risk of stroke and bleeding. The health care cost burden on AF patients using NOACs was substantial. Adherence to NOAC therapy was associated with a reduction in overall health care costs, since higher drug costs were offset by lower medical costs among adherent patients.

Our results provide valuable evidence regarding adherence and its related effect on total health care costs, which adds to our understanding of the risk-benefits of NOAC therapy and provides evidence for better decision-making processes by health care providers and managed care organizations. These findings underscore the need for encouraging and supporting patient adherence with NOAC therapy. Furthermore, the realworld estimates for drug and medical costs can be applied to future economic models, meta-analyses, indirect comparisons, and cost-effectiveness analyses. More studies that provide event-specific estimates (related to stroke, bleeding, deep vein thrombosis, and pulmonary embolism) of cost and adherence for newer NOAC drugs are warranted.

\section{Authors}

CHINMAY G. DESHPANDE, BPharm, MS, PhD; STEPHEN KOGUT, PhD, MBA; and CYNTHIA WILLEY, PhD, University of Rhode Island College of Pharmacy, Kingston.

AUTHOR CORRESPONDENCE: Chinmay G. Deshpande, BPharm, MS, PhD, 10301 Grosvenor Pl., North Bethesda, MD 20852.

E-mail: chinmay.cd@gmail.com.

\section{DISCLOSURES}

Funding to acquire the data source was provided by the University of Rhode Island College of Pharmacy, Kingston, to support PhD dissertation work. Deshpande is currently an employee of Pharmerit International.

\section{ACKNOWLEDGMENTS}

Data from the Optum Clinformatics Data Mart were accessed through the data server at the University of Rhode Island. The authors thank the data manager and the University of Rhode Island College of Pharmacy for their assistance.

\section{REFERENCES}

1. January CT, Wann LS, Alpert JS, et al. 2014 AHA/ACC/HRS guideline for the management of patients with atrial fibrillation: executive summary: a report of the American College of Cardiology/American Heart Association Task Force on practice guidelines and the Heart Rhythm Society. Circulation. 2014;130(23):2071-04.

2. Wolf PA, Abbott RD, Kannel WB. Atrial fibrillation as an independent risk factor for stroke: the Framingham Study. Stroke. 1991;22(8):983-88.

3. Roger VL, Go AS, Lloyd-Jones DM, et al. Heart disease and stroke statistics-2012 update: a report from the American Heart Association. Circulation. 2012;125(1):e2-e220.

4. Akin M, Schäfer A, Akin I, Widder J, Brehm M. Use of new oral anticoagulants in the treatment of venous thromboembolism and thrombotic prophylaxis. Cardiovasc Hematol Disord Drug Targets. 2015;15(2):92-96.

5. Seeger J, Bykov K, Bartels D, Huybrechts K, Zint K, Schneeweiss S. Safety and effectiveness of dabigatran and warfarin in routine care of patients with atrial fibrillation. Thromb Haemost. 2015;114(6):1277-89.

6. Yao X, Abraham NS, Alexander GC, et al. Effect of adherence to oral anticoagulants on risk of stroke and major bleeding among patients with atrial fibrillation. J Am Heart Assoc. 2016;5(2):e003074.

7. Beyer-Westendorf J, Ehlken B, Evers T. Real-world persistence and adherence to oral anticoagulation for stroke risk reduction in patients with atrial fibrillation. Europace. 2016;18(8):1150-57.

8. Coleman CI, Tangirala M, Evers T. Treatment persistence and discontinuation with rivaroxaban, dabigatran, and warfarin for stroke prevention in patients with non-valvular atrial fibrillation in the United States. PloS One. 2016;11(6):e0157769.

9. Zalesak M, Siu K, Francis K, et al. Higher persistence in newly diagnosed nonvalvular atrial fibrillation patients treated with dabigatran versus warfarin. Circulation. 2013;6(5):567-74

10. Laliberte F, Cloutier M, Crivera C, et al. Effect of rivaroxaban versus warfarin on health care costs among nonvalvular atrial fibrillation patients: observations from rivaroxaban users and matched warfarin users. Adv Ther 2015;32(3):216-27. 


\section{Real-World Health Care Costs Based on Medication Adherence and Risk of Stroke and Bleeding in Patients Treated with Novel Anticoagulant Therapy}

11. Biskupiak J, Ghate SR, Jiao T, Brixner D. Cost implications of formulary decisions on oral anticoagulants in nonvalvular atrial fibrillation. J Manag Care Pharm. 2013;19(9):789-98. Available at: https://www.jmcp.org/ doi/10.18553/jmcp.2013.19.9.789.

12. Chen J-Y, Zhang A-D, Lu H-Y, Guo J, Wang F-F, Li Z-C. CHADS2 versus $\mathrm{CHA}_{2} \mathrm{DS}_{2}$-VASc score in assessing the stroke and thromboembolism risk stratification in patients with atrial fibrillation: a systematic review and meta-analysis. J Geriatr Cardiol. 2013;10(3):258-66.

13. Camm A, Lip G, De Caterina R, et al. 2012 focused update of the ESC Guidelines for the management of atrial fibrillation: an update of the 2010 ESC Guidelines for the management of atrial fibrillation-developed with the special contribution of the European Heart Rhythm Association. Europace. 2012;14(10):1385-413.

14. Lane DA, Lip GY. Use of the CHA2DS2-VASc and HAS-BLED scores to aid decision making for thromboprophylaxis in nonvalvular atrial fibrillation. Circulation. 2012;126(7):860-65.

15. Ezekowitz MD, Wallentin L, Connolly SJ, et al. Dabigatran and warfarin in vitamin $\mathrm{K}$ antagonist-naive and experienced cohorts with atrial fibrillation. Circulation. 2010;122(22):2246-53.

16. Nau DP. Proportion of days covered (PDC) as a preferred method of measuring medication adherence. Pharmacy Quality Alliance. 2012. Available at: http://www.pqaalliance.org/images/uploads/files/PQA\%20 PDC\%20vs\%20\%20MPR.pdf. Accessed March 22, 2018.

17. Crivera C, Nelson WW, Bookhart B, et al. Pharmacy quality alliance measure: adherence to non-warfarin oral anticoagulant medications. Curr Med Res Opin. 2015;31(10):1889-95.

18. Charlson M, Szatrowski TP, Peterson J, Gold J. Validation of a combined comorbidity index. J Clin Epidemiol. 1994;47(11):1245-51.

19. Deyo RA, Cherkin DC, Ciol MA. Adapting a clinical comorbidity index for use with ICD-9-CM administrative databases. J Clin Epidemiol. 1992;45(6):613-19.

20. Dodd S, Bassi A, Bodger K, Williamson P. A comparison of multivariable regression models to analyse cost data. J Eval Clin Pract. 2006;12(1):76-86.

21. Brown JD, Shewale AR, Talbert JC. Adherence to rivaroxaban, dabigatran, and apixaban for stroke prevention in incident, treatment-naive nonvalvular atrial fibrillation. J Manag Care Spec Pharm. 2016;22(11):1319-29. Available at: https://www.jmcp.org/doi/10.18553/jmcp.2016.22.11.1319.

22. Gorst-Rasmussen A, Skjoth F, Larsen TB, Rasmussen LH, Lip GY, Lane DA. Dabigatran adherence in atrial fibrillation patients during the first year after diagnosis: a nationwide cohort study. J Thromb Haemost. 2015;13(4):495-504.

23. McHorney CA, Crivera C, Laliberté F, et al. Adherence to non-vitaminK-antagonist oral anticoagulant medications based on the Pharmacy Quality Alliance measure. Curr Med Res Opin. 2015;31(12):2167-73.
24. Shore S, Carey EP, Turakhia MP, et al. Adherence to dabigatran therapy and longitudinal patient outcomes: insights from the Veterans Health Administration. Am Heart J. 2014;167(6):810-17.

25. Beyer-Westendorf J, Forster K, Ebertz F, et al. Drug persistence with rivaroxaban therapy in atrial fibrillation patients-results from the Dresden noninterventional oral anticoagulation registry. Europace. 2015;17(4):530-38.

26. Fang MC, Go AS, Chang Y, et al. Warfarin discontinuation after starting warfarin for atrial fibrillation. Circulation. 2010;3(6):624-31.

27. Glader E-L, Sjölander M, Eriksson M, Lundberg M. Persistent use of secondary preventive drugs declines rapidly during the first 2 years after stroke. Stroke. 2010;41(2):397-401.

28. Kneeland PP, Fang MC. Current issues in patient adherence and persistence: focus on anticoagulants for the treatment and prevention of thromboembolism. Patient Prefer Adherence. 2010;4:51-60.

29. Francis K, Yu C, Alvrtsyan H, et al. Healthcare utilization and costs associated with dabigatran compared to warfarin treatment in newly diagnosed patients with non-valvular atrial fibrillation. Curr Med Res Opin. 2015;31(12):2189-95.

30. Bancroft T, Lim J, Wang C, Sander SD, Swindle JP. Health care resource utilization, costs, and persistence in patients newly diagnosed as having nonvalvular atrial fibrillation and newly treated with dabigatran versus warfarin in the United States. Clin Ther. 2016;38(3):545-556.el-6.

31. Fonseca E, Sander SD, Hess GP, Ghosh S. Hospital admissions, costs, and 30-day readmissions among newly diagnosed nonvalvular atrial fibrillation patients treated with dabigatran etexilate or warfarin. J Manag Care Spec Pharm. 2015;21(11):1039-53. Available at: https://www.jmcp.org/ doi/10.18553/jmcp.2015.21.11.1039.

32. Apostolakis S, Lane DA, Guo Y, Buller H, Lip GY. Performance of the HEMORR2HAGES, ATRIA, and HAS-BLED bleeding risk-prediction scores in nonwarfarin anticoagulated atrial fibrillation patients. J Am Coll Cardiol. 2013;61(3):386-87.

33. Fauchier L, Chaize G, Gaudin AF, Vainchtock A, Rushton-Smith SK, Cotté F-E. Predictive ability of HAS-BLED, HEMORR 2 HAGES, and ATRIA bleeding risk scores in patients with atrial fibrillation. A French nationwide cross-sectional study. Int J Cardiol. 2016;217:85-91.

34. Rothendler JA, Rose AJ, Reisman JI, Berlowitz DR, Kazis LE. Choices in the use of ICD-9 codes to identify stroke risk factors can affect the apparent population-level risk factor prevalence and distribution of $\mathrm{CHADS}_{2}$ scores. Am J Cardiovasc Dis. 2012;2(3):184-91

35. Mercaldi CJ, Siu K, Sander SD, et al. Long-term costs of ischemic stroke and major bleeding events among Medicare patients with nonvalvular atrial fibrillation. Cardiol Res Pract. 2012;2012:645469. 